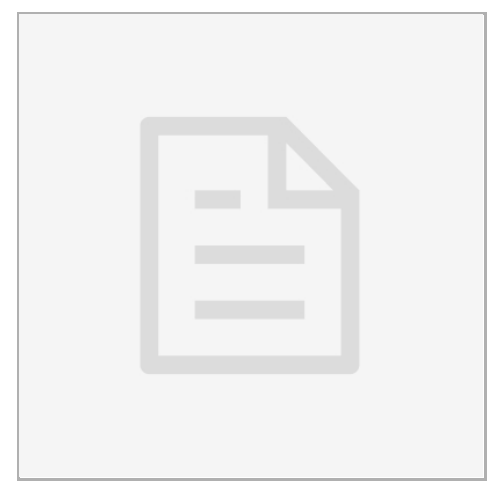

AUG 03, 2018

\section{open $\boldsymbol{\partial}$ Access}

DOI:

dx.doi.org/10.17504/protocol s.io.sazeaf6

Protocol Citation: Neha Kamat 2018. Kamat Lab Thin Film Hydration Protocol. protocols.io

https://dx.doi.org/10.17504/p rotocols.io.sazeaf6

License: This is an open access protocol distributed under the terms of the Creative Commons Attribution License, which permits unrestricted use, distribution, and reproduction in any medium, provided the original author and source are credited

\section{Protocol status: In} development We are still developing and optimizing this protocol

Created: Aug 02, 2018

Last Modified: Aug 03, 2018

PROTOCOL integer ID:

14393

\section{(3) Kamat Lab Thin Film Hydration Protocol}

Forked from a private protocol

Neha Kamat ${ }^{1}$

${ }^{1}$ Northwestern University

Build-a-Cell

Neha Kamat

\section{ATTACHMENTS}

\section{Lipid Film Hyration-Kamat}

Lab.docx

\title{
Assemble materials
}

Materials: 
Glass vials (any will do, but we use $2 \mathrm{~mL}$ Fisherbrand Class B Clear Glass Threaded vials: cat \#: 03339-21A)

Glass syringes of various sizes (ex. Hamilton gastight cat \#: 14-815-238, but any will do) for use with lipids and chloroform

Phosphate buffered saline 290 mOsm (Sigma, P4417-100TAB)

Mini Extruder (Avanti Polar Lipids)

Polycarbonate Membranes (Whatman Nuclepore Track-Etched Membranes, 19 mm)

Note

This is a general procedure to prepare phospholipid films with and without a Lissamine Rhodamine Membrane Dye to be hydrated later to prepare lipid vesicles. This procedure works best for studies with bulk small unilamellar vesicles.

\section{Final working conditions}

\section{Final working conditions}

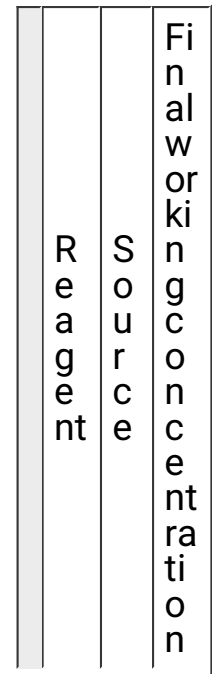




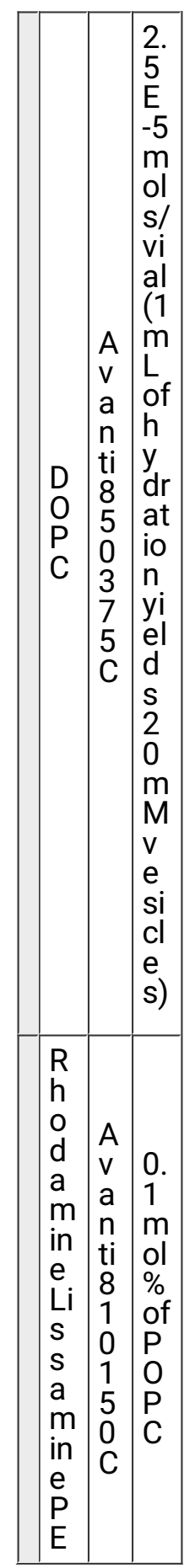

\section{Prepare lipid films in glass vials}

3 1. Determine the volume and molarity DOPC vesicles you want to create (ex: $1 \mathrm{~mL}$ of $20 \mathrm{mM}$ DOPC vesicles $=2.5 \mathrm{E}-5$ mols of DOPC. The stock of DOPC is $25 \mathrm{mg} / \mathrm{mL}$. To make giant, nice looking vesicles, it's best to prepare a more dilute sample of vesicles (like $200 \mathrm{uM}$ ). For small unilamellar vesicles, you can work with much higher concentrations of lipids (we can go up to $130 \mathrm{mM}$ for DOPC))

2. Add $2 \mathrm{E}-5$ mols DOPC and appropriate amount of Rhodamine PE into a glass vial since you are working with organic solvents. Cap and vortex briefly to ensure the two components are well 
mixed.

3. Remove cap and allow the chloroform to evaporate away in the hood.

4. Place the vial into a vacuum chamber for $>1 \mathrm{hrs}$ or leave overnight in order to remove all residual traces of solvent.

5. Cap and store vials in freezer until ready to be used and hydrate with $1 \mathrm{~mL}$ of aqueous hydration buffer when you are ready to prepare vesicles.

\section{Hydrate films and extrude vesicles}

4 - Add $1 \mathrm{~mL}$ of PBS to each vial, place in $60^{\circ} \mathrm{C}$ oven for $1 \mathrm{hr}$, then vortex 10 seconds. Lipids should easily assemble. If clumps or aggregates persist after several vortexing attempts, this is often a sign something went wrong in vesicle assembly. The heating step is not necessary and can be excluded, but helps when other membrane components are involved like diblock copolymers.

- Extrude films at room temperature through $100 \mathrm{~nm}$ polycarbonate membranes using a mini extruder and heating block described on the Avanti website (7 passes through membrane is fine, $9-11$ is better, more than that is unnecessary according to my post extrusion analysis of vesicle size distribution on DLS.)

\section{Purify vesicles if hydration buffer contained a dye or solute.}

5 Set up columns (BioRad Poly Prep 7311550) containing 6 mLs of Sepharose 4B (size exclusion column media). Purification should be conducted on the same day as a subsequent assay. 